\title{
Correspondence
}

\section{Optical Channels: Practical Limits with Photon Counting}

\section{J. R. PIERCE}

Abstract-In optical communication, ideal amplification of the received signal leads to a limiting signaling rate of 1 nat per photon. This is much inferior to the optimum limit of $k T$ joules/nat, which we can theoretically approach by counting photons. Practically, the rates we can attain by photon counting will be limited by how elaborate codes we can instrument rather than by thermal photons.

Consider a free-space path such as we might have between space vehicles. For a wavelength $\lambda$, a distance $L$ and transmitting antennas of effective areas $A_{T}$ and $A_{R}$ the ratio of received power $P_{R}$ to transmitted power $P_{T}$ is ${ }^{1}$

$$
P_{R} / P_{T}=A_{T} A_{R} / \lambda^{2} L^{2}
$$

This suggests the use of a short wavelength.

Going to optical wavelengths requires very smooth antenna surfaces and very precise pointing. Further, at optical frequencies we encounter quantum effects. Here we disregard antenna and pointing problems and consider how quantum effects will limit a communication system.

In receiving a signal mixed with Johnson noise, we have the option of amplifying the signal with an ideal amplifier of power gain $G$ and bandwidth $B$. The Gaussian noise power $P_{n}$ in the output of the amplifier will be $e^{2,3,6}$

$$
P_{n}=\frac{h f}{e^{h f / h T-1}} B+(G-1) h f B .
$$

When $h f \ll k T$, the noise power density at the input of the amplifier is nearly $k T$. According to Shannon ${ }^{4,5}$ when noise of this power density is added to a signal, the limiting information rate $R$ in nats per joule of transmitted power, which is attained as $B$ approaches infinity, is

$$
R=1 / k T \text { nats } / \text { joule. }
$$

When $h f \gg k T$ the second term on the right of (2) dominates. We see that this second term is not amplified noise because $h f B$ is multiplied by $(G-1)$, not $G$. However, when the gain $G$ is very large, the second term is very nearly equal to a Gaussian noise density $h f$ multiplied by the gain $G$. Thus, following Shannon the limiting information rate in nats per joule of transmitted power will be

$$
R=1 / h f \text { nats } / \text { joule. }
$$

The energy per photon is $h f$. Thus, the limiting rate given by (4) is 1 nat per photon. This limit holds only if we amplify

Paper approved by the Editor for Communication Theory of the IEEE Communications Society for publication without oral presentation. Manuscript received April 7, 1978; revised June 14, 1978.

The author is with the Department of Electrical Engineering, California Institute of Technology, Pasadena, CA 91125.

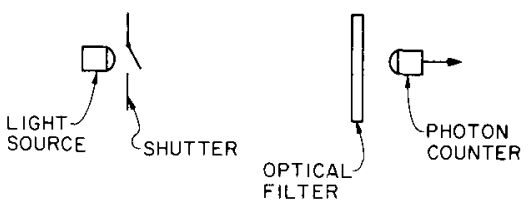

Figure 1. A Photon Channel. A light source transmits photons during intervals when the shutter is open. Received signal and thermal photons pass through an optical filter intended to eliminate out-ofband thermal photons. Each photon that passes the optical filter is counted by a photon counter. In a real system, the light source and shutter could be replaced by a pulsed laser.

the received signal with an ideal amplifier. It does not hold for simple photon counting or for various other forms of transmission and reception. ${ }^{6-9}$

Indeed, if no noise is added to the signal, the number of nats we can transmit per photon is unbounded. ${ }^{6-9}$ We can see this by a very simple argument.

Consider the signaling system shown in Figure 1. The transmitter consists of a light source and a shutter.* The receiver consists of an optical filter and a photon counter, which we will assume to emit a pulse when one or more photons strike it. The purpose of the optical filter is to exclude thermal photons. Initially, we will assume that there are no thermal photons and we will dispense with the optical filter.

In signaling, we assume a code word whose length is $N$ time intervals, each of duration $t$. Each of these code words has a "pulse" in one time interval only, so that the form of signaling is quantized pulse position modulation.

In signaling, we open the shutter during only one time interval out of the $N$ in the code word. If we impose no bandwidth limitation in the transmission path, so that classically the path has a constant loss and delay, we can never receive a photon during any time interval except the one corresponding to the time interval during which the shutter was open**.

Let us assume that the average number of photons received in the time interval corresponding to the opening of the shutter is $M$. If $M$ is fairly large, we will almost always receive at least one photon, and thus we will almost always receive a proper code word. The information per code word will then be

$\ln N$ nats.

The average number of photons per code word is

$M$ photons.

Hence, the number of nats per photon is

$(\ln N) / M$ nats/photon.

* In a "practical" system we could send a pulse by pulsing a semiconductor laser rather than by opening a shutter.

** One can quibble here or later that $P_{R} / P_{T}$ in (1) changes with frequency or that propagation near the source may be slightly dispersive, or that the actual energy of the emitted photon cannot be known. But, for a signaling rate far less than the frequency of the light used, transmission is practically the same for all frequency components of the signal, and the fractional range in photon energies is extremely small. 
By making $N$ large, we can make the information rate in nats/photon as large as we wish. But growth is only as the logarithm of $N$, and is impractical to make $\ln N$ very large.

What about the effects of thermal photons in limiting the rate of signaling? Oddly enough, the theoretical limit for very small signal strength and unlimited bandwidth is the classical limit given by (3), that is, $k T$ joules/nat. ${ }^{6,7} \mathrm{I}$ have included in Appendix $\mathrm{A}$ a simple demonstration that for a coherent signal source, $k T$ joules/nat can be approached when $h f \gg k T$.

Let us now ask, how many nats per photon do we need in order to attain the limiting $k T$ joules/nat?

A photon has an energy $h f$. If we require $k T$ joules/nat, the number $Q$ of nats per photon must be

$$
Q=h f / k T=4.80 \times 10^{-11}(f / T) \text { nats/photon }
$$

How big will $Q$ be for optical frequencies and low temperatures? Let us assume a wavelength of $5,000 \AA$, corresponding to a frequency of $6 \times 10^{14} \mathrm{~Hz}$, and a temperature of $6 \mathrm{~K}$. For these values,

$$
Q=4,800 \text { nats } / \text { photon }
$$

If we tried to attain this by means of quantized pulse position modulation, length $N$ of our code words would have to be at least

$$
N=e^{4,800} \text {. }
$$

This, of course, is ridiculous.

The practical conclusion is that in optical signaling at low temperatures we encounter insuperable problems of encoding long before we approach the theoretical limit of $k T$ joules/nat.

This seems plausible from another point of view. At what rate do we receive thermal photons? $P_{n}$ of (2) gives the thermal photon power in one mode of propagation if we set $G=1$. The rate $p$ at which we receive thermal photons in a coherent (one-mode) system is thus

$$
p=\left(e^{h f / k T}-1\right)^{-1} B \text { photons/second }
$$

Let us set $B=1$, which allows time intervals $t$ of around $1 \mathrm{~s}$. For $B=1, f=6 \times 10^{14}$ and $T=6$,

$$
p=e^{-4,800} \text {. }
$$

This is almost no noise photons per time interval.

With codes of any reasonable length and elaborateness, we will fall far short of $k T$ joules/nat, so far short that we can afford to ignore thermal photons. For optical frequencies and low temperatures, the rate at which we can signal, measured in nats per photon, will be limited by our ability to implement codes, not by thermal photons.

\section{APPENDIX}

Here we will consider a single-mode photon communication system in which there is a noise source of photons with an average of $n_{0}$ photons per time interval, and a signal source of photons with an average of $n$ photons per time interval when the transmitter is on (when the shutter is open).
According to Shannon, ${ }^{4}$ the rate of transmission $R$ measured in nats per symbol (that is, per time interval) is

$$
R=H(Y)-H(Y \mid X)
$$

Here $X$ represents shutter position $(0=$ closed; $1=$ open $)$ and $Y$ represents photons received ( $0=$ none; $1=1$ or more). $R$ is a function of $n, n_{0}$ and the probability $\alpha$ that the shutter will be open.

In order to compute $H(Y)$ we need to know the probabilities $p(0)$ (that will receive no photons in an interval) and $p(1)$ (that will receive some photons in an interval), assuming that we have no knowledge of $X$. We see that

$$
\begin{aligned}
& p(0)=\alpha p(0 \mid 1)+(1-\alpha) p(0 \mid 0) \\
& p(1)=1-p(0) .
\end{aligned}
$$

It turns out that the number of nats per photon is greatest when the number $n$ of signal photons and the number $n_{0}$ of noise photons are much smaller than unity. When this is so, we can use the following approximate expressions for the case in which the shutter is closed, so that there are thermal photons only, and no signal photons

$$
\begin{aligned}
& p(0 \mid 0)=1-n_{0} \\
& p(1 \mid 0)=n_{0} .
\end{aligned}
$$

We have no expression for the case in which the shutter is open and we receive both coherent signal photons and noise photons. However, if we assume that we will receive more photons than the number of signal photons alone, we will underestimate $R$ by disregarding the effect of noise photons when the shutter is open and taking

$$
\begin{aligned}
& p(0 \mid 1)=1-n \\
& p(1 \mid 1)=n .
\end{aligned}
$$

Making these assumptions, and assuming that $n_{0} \ll n$ and $\alpha \ll 1$, we find

$$
\frac{R}{\alpha n}=-\ln n_{0} .
$$

This is the number of nats per photon transmitted. Equation (A-6) leads to the classical limit of $k T$ joules/nat when the frequency is high enough so that quantum effects are very strong.

From (2) we see that for a single-mode transmission system, when $h f \gg k T$ the number $n_{0}$ of thermal photons in a time $t$ can be taken as

$$
n_{0}=B t e^{-h f / k T}
$$

From (A-6) and (A-7)

$$
\frac{R}{\alpha n}=\frac{h f}{k T}-\ln B t .
$$

Because we have assumed that $h f \gg k T$, the first term on the right is much larger than unity. What about $\ln B t$ ?

$B$ is really a sort of mean bandwidth of the optical filter in Fig. 1. This must be made wide enough so as not to lose many 
signal photons when the shutter is open, but narrow enough not to let in too many noise photons. This means that $B t$ should be around unity, but probably somewhat larger.

Thus, when quantum effects are most pronounced, we can disregard the second term in (A-9). Then

$$
\frac{R}{\alpha n}=\frac{h f}{k T} \text { nats } / \text { photon. }
$$

This corresponds to

$$
\frac{1}{k T} \text { nats/joule. }
$$

Gordon $^{6}$ makes a somewhat similar calculation of channel capacity but does not carry the argument to (A-10) above. Equation (A-10) appears to be implicit in Helstrom et al but is not stated explicitly.

\section{ACKNOWLEDGMENT}

The author wishes to thank J. P. Gordon, Carl W. Helstrom and Jon Mathews for helpful correspondence and comments.

\section{REFERENCES}

1. Schelkunoff, S. A. and Friis, H. T., Antenna Theory and Practice, Wiley and Sons, New York, 1952, p. 43.

2. Heffner, H., The Fundamental Noise Limit for Linear Amplifiers, Proc. IRE, 50, 1604-1608, 1962.

3. Gordon, J. P., Louisell, W. H., and Walker, L. R., Quantum Fluctuations and Noise in Parametric Processes, II, Phys. Rev., 129, January, 1963, pp. 481-485.

4. Shannon, C. E. and Weaver, W., The Mathematical Theory of Communication, The University of Illinois Press, 1959.

5. Pierce, J. R., Symbols, Signals and Noise, Harper and Row, 1961 Chapter 10.

6. Gordon, J. P., Quantum Effects in Communication Systems, Proc. Inst. Radio Eng., 50, 1898-1908, September, 1962.

7. Helstrom, C. W., Liu, J. W. S., and Gordon, P., Quantum Mechanical Communication Theory, Proc. IEEE, 58, October, 1970, pp. 1578-1598.

8. Helstrom, C. W., Capacity of the Pure-State Quantum Channel, Proc. IEEE, 62, January, 1974, pp. 140-141.

9. Yuen, Horace P., Kennedy, Robert S. and Lax, Melvin, Optimum Testing of Multiple Hypotheses in Quantum Detection Theory, IEEE Trans. Information Theory, IT-21, March, 1975, pp. 125134.

\section{Combinatorial Issues in Mobile Packet Radio Networks}

\section{DANIEL MINOLI AND ISRAEL GITMAN}

\begin{abstract}
A Packet Radio Network is a store-and-forward packet system employing radio links. A Mobile Packet Radio Network generalizes this system by allowing every radio unit to be independently

Paper approved by the Editor for Computer Communication of the IEEE Communications Society for publication after presentation at the 28th Vehicular Technology Conference, Denver, CO, 1978. Manuscript received August 3,1976; revised May 2, 1978.

D. Minoli was with Network Analysis Corporation, Great Neck, NY 11024. He is now with Bell Laboratories, Holmdel, NJ 07733.

I. Gitman is with Network Analysis Corporation, Great Neck, NY 11024.
\end{abstract}

in motion. In this paper a model for the analysis of connectivity as a function of time in such a network is presented and used to solve for parameters of interest. The model and the related analysis are guides for developing procedures for monitoring connectivity.

\section{INTRODUCTION}

A Packet Radio Network (PRNET) is a store-and-forward packet switching radio system.1,2 Functionally, it includes three types of devices: Terminal, Repeater, and Station. A repeater provides area coverage for mobile terminals and also acts as a relay node. The station provides global control functions, gateway functions for interfacing with other networks, and initialization functions. A centralized hierarchical routing algorithm is assumed in this paper. ${ }^{3}$ According to this algorithm all packet transmissions between nodes in the network are routed via the station. Furthermore, the station initializes and periodically updates repeater parameters for routing.

One of the objectives of the system is to enable communication between mobile devices. Up to the present, investigations have been limited to the study of Stationary Packet Radio Networks, which are partially characterized by the following two conditions:

1. Terminals, repeaters and stations are stationary.

2. The area in which terminals originate is fixed and is covered by appropriately placing repeaters and stations at some initial time $t_{o}$.

On these assumptions, routing algorithms and initialization algorithms have been developed.

The Mobile Packet Radio System generalizes the stationary system by allowing every element of the hardware to be independently in motion. The advantages and applicability of this system arises from its capability of providing communications between mobile terminals (autos, ships, hand-held devices, etc.) via mobile repeaters on vans, ships or airplanes to a (potentially mobile) computer center or monitoring station. A repeater differs from a terminal, in that it is not a source or sink for information flow, and its objective is to extend the effective communication range of terminals (to achieve wide coverage). Consequently, a mobile radio system would typically consist of a large collection of mobile terminals and a small number of repeaters; the mobility of the repeaters may be dictated by the application (e.g., a fleet of ships). Mobile radio systems have been available for many years; however, the principal mode of communication has mostly been through dedicated or reserved resources without resource sharing; hence, spectrum was not efficiently utilized. The Packet Radio System, on the other hand, is a multiple access random transmission scheme.

There is a clear distinction between the two systems, not only in the definition and analysis, but also the problems one must address. For example, in a Packet Radio Network (PRNET) one is trying to determine the location of repeaters to achieve optimal coverage of a certain area. A repeater may be permanently placed in a precalculated location. In the Mobile Packet Radio Network (MPRNET) repeaters are assumed in motion.

In PRNET, a particular and predetermined zone is covered.

In MPRNET, the covered zone varies with time. Requirements as to the coverage of a particular area, may, however, be imposed.

In PRNET, once the system is initialized, it will remain so, unless there is a high failure rate of repeaters. 